Voix et Images

voixetimages

\title{
Notes bibliographiques
}

\section{Bernard Andrès, Jean-Guy Nadeau, Monique Herbeuval et Gilles Thérien}

Volume 7, numéro 1, automne 1981

\section{Adrien Thério}

URI : https://id.erudit.org/iderudit/200317ar

DOI : https://doi.org/10.7202/200317ar

Aller au sommaire du numéro

Éditeur(s)

Les Presses de l'Université du Québec

ISSN

0318-9201 (imprimé)

1705-933X (numérique)

Découvrir la revue

Citer ce compte rendu

Andrès, B., Nadeau, J.-G., Herbeuval, M. \& Thérien, G. (1981). Compte rendu de [Notes bibliographiques]. Voix et Images, 7(1), 201-206.

https://doi.org/10.7202/200317ar d'utilisation que vous pouvez consulter en ligne.

https://apropos.erudit.org/fr/usagers/politique-dutilisation/ 


\section{Notes bibliographiques}

(rédigées par Bernard Andrès, Jean-Guy Nadeau, Monique Herbeuval et Gilles Thérien)

\section{REVUES}

\section{The American Review of Canadian Studies}

volume $X$, number 2, Autumn 1980, Department of History, University of Vermont, Burlington, Vermont 05405

Sommaire: Robert Lecker "Quantum physics and nouveau roman: The New Ancestors», Richard S. Sorrell «Jack Kerouac, French Canada, and France»Donald Alper «Congressional attitudes toward Canada and Canada-United States relations», Don Munton «Simulating Canadian-American negociations: A boundary waters example», Robert $M$. Gill «Bilingualism in New Brunswick and the future of I'Acadie», Edward G. Grabb and James W. Rinehart «A research note on class and authoritarian perspectives in Canada", André Sénécal "Quebec studies: A guide to the bibliographies".

M.H.

\section{Bulletin du Centre d'études franco-canadiennes de l'Ouest}

$n^{\circ}$ 7, février 1981. 200 avenue de la Cathédrale, Saint-Boniface, Manitoba $\mathrm{R} 2 \mathrm{H} \mathrm{OH7.}$

Un éditorial de A. Fauchon. La première partie d'une étude historique sur les Canadiens français de St-Paul et Minneapolis par Rossel Vien. Des comptes rendus de «Derrière les barbelés des Nazis", "Le Roitelet " et "Les Français dans l'Ouest canadien". L'inauguration de la salle Robert-Painchaud (Annette Saint-Pierre) et l'annonce d'un colloque au CEFCO.

M.H.

n० 8, mai 1981.

Un éditorial de Annette Saint-Pierre. La seconde partie de l'étude historique présentée par Rossel Vien (Une colonie canadienne française au Minnesota). Un article de Émile Couture sur les conventum des années 1920-21. Le travail des documentalistes au CEFCO. Un article de J. Raimbault sur le journal Le Métis. Des comptes rendus de publications faites à Saint-Boniface. 


\section{Études littéraires}

volume 13, n 1, avril 1980. PUL, C.P. 2447, Québec G1K 7R4.

Ce numéro est entièrement consacré au problème du récit au cinéma. On y trouve une bonne variété d'articles, tantôt de théorie, tantôt d'analyse. En ce sens, le numéro est représentatif du travail qui se fait au sujet du cinéma. Le seul reproche que l'on peut adresser à ce numéro, c'est justement d'être très classique, voire conservateur, dans son approche du récit au cinéma. On ne s'écarte guère de la pensée issue des Saussure, Jakobson, Greimas, Genette. Une bibliographie aurait été aussi bienvenue et utile à tous les lecteurs que le sujet intéresse.

G.T.

\section{La nouvelle Barre du jour}

n० 98, janvier 1981, Succ. Outremont, Outremont, Québec H2V 4M8.

Des fictions de Louise Dupré et Danielle Fournier, des photographismes et des photographies de Danielle Péret et Pierre Chicoine, un essai de Richard Boutin "... mander une photo à...", enfin des commentaires de Claude Beausoleil et Anne-Marie Alonzo.

M.H.

n० 99, février 1981

Des fictions de Claude Paré, Rachel Leclercq, Charles Deschênes, Claudine Potvin, Danielle Dussault, Yvon Boucher et Renée Berthe-Drapeau dont on remarque "La Lettre ingrate». Un essai de François Charron "La Création du monde* en hommage à Robert Savoie et des commentaires de Philippe Haeck, François Vasseur, André Beaudet et Claude Beausoleil.

M.H.

$n \circ$ 100-101, mars 1981

Avec les signatures de trente poètes, la *cavalière NBJ» célèbre son centième numéro entièrement consacré à la poésie québécoise. En complément des notes bibliographiques. Un beau recueil ce Poésie 19811

M.H.

$\mathrm{n}^{\circ}$ 102, avril 1981

La Femme et la Ville: treize femmes nous parlent de leur vision de la ville et nous entraînent dans leur vertige écrit. Ont participé à ce numéro: Nicole Brossard, Jovette Marchessault, Gail Scott, France Théorêt, Germaine Beaulieu, Yolande Villemaire, Marie-Claire Blais, Pauline Harvey, Marie Lafleur, Louky Bersianik, Suzanne de Lotbinière-Harwood, Régine Robin et Josée Yvon.

M.H.

\section{Estuaire}

n० 19, printemps 1981. C.P. 828, Haute-Ville, Québec G1R 4 S7.

Des poèmes de J. Izoard, M. Uguay, P.-L. Tetreault, S. Turcotte, M. Quaghebeur, G.-R. Archambault, M. Leclerc. Des chroniques de C. Bouchard, P. Haeck, C. Robitaille et F. Vasseur.

M.H.

\section{Revue d'ethnologie du Québec}

$\mathrm{n}^{\circ} 11$, novembre 1980. Éditions Leméac, 5111, avenue Durocher, Montréal H2V $3 \times 7$.

Au sommaire: «Le marteau à débotter» par Robert-Lionel Séguin, “L'affaire Blanche Garneau» par Réal Bertrand, «Poterie d'hier et d'aujourd'hui »par Andrée Lapointe, "La danse chez les Indiens de la Nouvelle-France au XVIle et XVIIle 
siècles"par Jean-Paul Massicotte et Claude Lessard et «La maison Bernatchez à Saint-Thomas de Montmagny» par Anne Bernatchez.

M.H.

$\mathrm{n}^{\circ} 12$, décembre 1980

Ce numéro est essentiellement consacré aux «Façons de parler proverbiales, triviales, figurées, des Canadiens au XVIIle siècle" du Père Potier tandis que Robert-Lionel Séguin se demande si le fléché québécois est d'origine française et que Jeanne-Morin traite de la représentation des nomades et sédentaires dans la littérature québécoise.

M.H.

\section{Liberté}

n० 133, janvier-février 1981, C.P. 399, succ. Outremont, Montréal, Québec, H2V 4N3.

Dans ce numéro il est question de l'usage politique du pronom nous dans les discours par Pierre Laurette. Une nouvelle polémique?: Jean-Marcel Paquette et François Ricard critiquent sévèrement le territoire imaginaire de la culture. Un texte émouvant et simple: I'exil de M.-C. Blais, de l'humour acidulé avec Bernard Lévy et des poèmes de Juan Garcia, Pierre Des Ruisseaux et René Lapierre. De nombreuses chroniques dont l'éloge des œuvres primées: Les portes de Gubbio et La vie en prose ainsi que de la difficulté de représenter l"écrivain au cinéma. M.H.

n० 134, mars-avril 1981

Liberté se pose des questions sur l'institution littéraire québécoise offrant des textes de Gilles Marcotte, André Belleau, Jean Larose, François Ricard, Jacques Godbout, François Hébert, Pierre Nepveu, René Lapierre, Yvon Rivard, Réjean Beaudoin, Gilles Archambault, Marie-José Thériault, Robert Mélançon et Vital Gadbois. On nous livre les résultats d'une enquête menée sur les différents aspects de l'institution littéraire auprès d'une quarantaine d'écrivains. Enfin, pour terminer les chroniques habituelles.

M.H.

\section{Le temps fou}

$n^{\circ} 13$, février-mars 1981, 4073, rue Saint-Hubert, Montréal, H2L 4A7.

Un portrait de Claude Ryan par Pierre Vallières, un dossier sur l'Agence de presse libre au Québec par Louis Fournier, un article de Louise Vandelac sur la contraception. La vie et l'œuvre de Violette Leduc, le régime révolutionnaire au Nicaragua. Des chorniques sur l'écologie, les femmes, l'économie, le travail, la musique, le cinéma, les livres.

M.H.

$n^{\circ} 14$, avril-mai 1981

Un débat sur le regroupement des autonomies. De nombreux articles sur un peu de tout (média, histoire, économie, éducation et sexualité). Un dossier sur le chasseur $F-18$, des nouvelles internationales et enfin des comptes rendus de livres, de revues et de disques.

M.H.

Jeu

n० 13, automne 1979, Case postale 1600/E, Montréal, H2T 3B1.

Dossier sur Jean-Claude Germain, par G. David, F. Noël, N. Audet, L. Mailhot, E. Bessette et $P$. Lavoie (qui a signé la bio-bibliographie, pp. 105 à 141); « Jeu après 4 ans..., par G. David; ; cle comité du 13 septembren, par le collectif; les chroniques de spectacles et de publications, informations et mises au point. 
n० 14, 1980.1, lbid.

"Vers des États généraux du théâtre professionnel », par G. David et D. Journault ; des articles sur le C.E.A.D., le Patriote, la censure à la C.E.C.M., Avignon 1979, une page... blanche sur la Place des Arts; l'Eskabel, les scénographes actuels, les Gens d'en bas, le Theâtre des confettis. Bernadette et Juliette, et les chroniques habituelles.

B.A.

n० 15, 1980.2, lbid.

Historique de I'ACTA/AQJT (1958-1980), un dossier volumineux sur le jeune théâtre, par G. David, G. Beaulne, H. Beauchamp, M.H. Falcon, M.C. Larocque, J.G. Sabourin et P. Rousseau. Entretien de L. Hébert avec H. Dupuis, sur I'animation théâtrale, et les chroniques.

B.A.

no 16, 1980.3, lbid.

Numéro spécial «Théâtre-Femmes"; une soixantaine de collaboratrices. Entretiens, témoignages, analyses, témoignant de la vigueur du mouvement féministe dans le champ theâtral et culturel. Un très beau et bon numéro qui fera date, illustré notamment par Maureen Maxwell. A noter en guise de conclusion, un «éditorial en forme de test " qui révèlera "où vous en êtes avec la question du féminisme».

B.A.

n० 17, 1980.4, Ibid.

Prolonge le $n^{\circ}$ précédent avec un dossier sur Louisette Dussault, mais aussi: le théâtre à l'étranger (Japon, France, Berlin-Ouest, théâtre gai à San Francisco), un article de J. Féral sur le Carnaval et d'autres sur la marionnette, différents festivals, colloques et conférences, sur le théâtre de l'opprimé, etc. Chroniques.

B.A.

$n^{\circ} 18,1981.1$, lbid.

Sixième année de publication! A noter le dossier de $C$. Hébert sur le Burlesque à Montréal (La Poune, Juliette Pétrie, Jean Grimaldi, Le Nouveau Théâtre National, Les Variétés, etc.). et deux autres sur le Trident, à Québec, et sur les Mimes Omnibus. Chroniques habituelles.

B.A.

\section{Répertoire théâtral du Québec 1981}

édition Jeu.

Après le Répertoire 1979-80, ce dernier volume, sous la responsabilité de G. David, reprend, complète et amplifie la somme de renseignements divers sur les troupes et théâtres du Québec, hors-Québec, cafés-théâtres, théâtres d'été, classement par régions, organismes subventionneurs, bilan des subventions 1979-80 (par A. Gruslin), associations professionnelles, institutions d'enseignement et de recherche, publications, mass-médias, organismes internationaux et festivals. Suivi d'un index fort pratique.

B.A.

\section{ROMANS}

\section{Menfou Carcajou}

de Suzanne Martel, Montréal, Leméac, coll. roman québécois, 1980. Première partie: Ville-Marie, 254 p.; Deuxième partie, la baie du Nord, 203 p. 
Dans la première partie du récit, ce sont les habitants de l'île de Montréal au XVIlème siècle que Suzanne Martel fait habilement revivre. Issue d'un sérieux travail de documentation, l'authenticité de leur vie ne porte aucun préjudice à la part du romanesque. La signature d'un contrat de mariage, la cueillette des noix le dimanche, la journée des chandelles et la peine du pilori sont l'occasion de faire naitre un monde fictif attachant.

Se détache la figure de Menfou Carcajou, ce coureur des bois empreint des coutumes iroquoises, dont le destin mouvementé nous emmène, dans la seconde partie du récit, jusqu'à la baie du nord que les Français veulent reprendre aux Anglais. L'expédition du Chevalier de Troyes, dans laquelle s'est engagé le coureur des bois, est fertile en péripéties qui sont rendues avec humour. Après six mois d'une captivité hallucinante sur un bateau anglais prisonnier des glaces, Menfou, de retour à Ville-Marie, s'aperçoit qu'il est trop tard pour gagner le cœur de sa belle. Malgré l'amitié de sa jeune protégée. Sophie, «le vagabonơ des forêts" retourne alors vers ses frères les Iroquois. Le plaisir de la lecture nous a conduit tout droit au cœeur de ce XVIlème siècle qui nous paraissait si lointain!

M.H.

\section{La Corriveau}

de Andrée Lebel, Montréal, Libre Expression, 1981, 206 p.

Roman historique qui tente de réhabiliter Marie-Josephte Corriveau que la légende présente comme une sorcière ayant assassiné ses deux maris. Le récit insiste beaucoup sur le poids du régime militaire britannique au Québec en 1763 ainsi que sur la lâcheté des Québécois dont les commérages contribuèrent à la perte de la Corriveau.

M.H.

\section{Le P'tit ministre-les-pommes}

de Réal-Gabriel Bujold, Montréal, Leméac, 1980.

Un récit qui évoque une année (1954-55) dans la vie d'une petite paroisse de la Baie des Chaleurs: jeux d'enfants et de grandes personnes, jeux de l'amour et du pouvoir: un petit monde reconstitué avec beaucoup d'humour, mais dans un texte difficile à lire, inutilement compliqué de ruptures syntaxiques et de métaphores incohérentes.

J.-G. N.

\section{LE THÉĀTRE QU'ON PUBLIE}

\section{Une amie d'enfance}

de Louise Roy et Louis Saia. Théâtre/Leméac, n 89, 1980.

Préface de Laurent Maithot. Garden-Party à Laval. Deux jeunes couples de banlieue passent la soirée autour d'un piètre repas. Échange de propos dont la banalité finit par s'estomper à mesure que croit une certaine tension dramatique, toujours détendue par l'humour des dialogues, plus savoureux que le jello du dessert. Excellente caricature du afaubourg-way-of-life». 


\section{La trousse}

de Louis-Marie Dansereau. Théâtre/Leméac, $n^{\circ}$ 90, 1980.

Préface de Doris-Michel Montpetit. Ėtre une "trousse", c'est "vivre de son cul». Monologue d'une femme seule à la recherche d'une différence et d'une identité, d'un nom: Minoune. Haut en couleurs (et en photos du spectacle). Cette quête s'inscrit dans un langage cru et humoristique. L'auteur parvient-il à se départir de l'influence de Tremblay?

\section{Les vaches sont de braves types (suivi de trois autres pièces)}

de Jean Gagnon, Théâtre/Leméac, $n^{\circ}$ 91, 1981.

Courtes pièces de théâtre radiophonique, pour sourire sans prétention. Préface de Gilles des Marchais. Jean-Baptiste Poupinot, insecte domestique: métamorphose d'un vieux célibataire en coquerelle, à la suite d'un complot de village contre le marginal; Les vaches sont de braves types: dialogue d'un fonctionnaire avec un bovin de Lotbinière, en vue de la constitution d'un mouvement de libération... des vaches (!): De gauche à droite, en allant vers le bas (...): cours du soir en gestion commerciale. L'éditeur, la mercière, M. Meuble et le Roi du TV apprennent ensemble à se détester avant de faire... faillite.

B.A.

\section{Isabelle}

de Pierre Dagenais. Théâtre/Leméac, $n^{\circ}$ 92, 1981.

Préface de Gilles Desmarchais. Drame psychologique sur le problème philosophique de la vérité, du mensonge... et du silence. Écrit dans une langue soutenue par le fondateur du Théâtre de l'Équipe (1942). Un discours dramatique dont on a un peu perdu l'habitude. Un journaliste, un peintre et un libraire tentent d'expliquer la tragédie d'un suicide causé par une femme énigmatique: Isabelle. Jeu de la vérité et de l'hypocrisie sociales, où le préfacier voit une dramaturgie "au-delà de la dramaturgie pure et simple", une référence à l'homme «éternel " et au Corneille du Cid...

\section{Un reel ben beau, ben triste (suivi de quatre autres pièces)}

de Jeanne-Mance Delisle. Théâtre/Les éditions de la pleine Lune, 1980.

Cinq pièces qui mettent en scène la solitude de chaque être et surtout de la femme en butte au pouvoir oppressif de l'homme. Contes, légendes, sorcellerie sont convoqués (quel accord!) à un reel ben beau, ben triste. Drame naturaliste en Abitibi. Lancinant, «le reel du pendu " joué par Ti-fou, l'arriéré-mental et meurtrier de sa scur, scande la révolte des personnages.

\section{MONOLOGUES}

La vie... des fois

de Denise Guénette. Montréal, Éditions de la pleine Lune, 1980, 139 pages.

Un regard féroce mais plein d'humour sur la vie quotidienne. A lire sans préjugés! 